\title{
Diverticulite Aguda Complicada Tratada por Cirurgia Laparoscópica Assistida Com a Mão (Hals) - Descrição da Técnica e Revisão da Literatura
}

\author{
Complicated Diverticulitis Operated With the Hand Assisted Approach \\ (Hals) - Description and Literature Review
}

\author{
GUINES ANTUNES ALVAREZ ${ }^{1,2}$; MÔNICAMAZZURANA ${ }^{1}$ \\ ${ }^{1}$ Serviço de Cirurgia Digestiva do Hospital Beneficência Portuguesa de Santos, Santos, SP, Brasil; ${ }^{2}$ Faculdade de \\ Ciências Médicas de Santos - SP - Brasil.
}

ALVAREZ GA; MAZZURANA M. Diverticulite Aguda Complicada Tratada por Cirurgia Laparoscópica Assistida com a Mão (Hals) Rev bras Coloproct, 2006;26(3): 275-279.

\begin{abstract}
RESUMO: Introdução: A doença diverticular é freqüente em nosso meio e o tratamento clínico é suficiente para a grande maioria dos casos. No entanto, o tratamento cirúrgico fica reservado para as formas complicadas da doença, para o insucesso da terapia clínica e nos casos de imunossupressão. A cirurgia laparoscópica vem ganhando espaço como modalidade terapêutica na doença diverticular, diminuindo o tempo de internação e melhorando o resultado cosmético e funcional apesar de algumas dificuldades inerentes ao método. Objetivo: O objetivo dos autores é descrever a técnica de cirurgia laparoscópica assistida com a mão em dois casos de diverticulite complicada (um caso de fístula colo-vesical e outro de abscesso) e rever a literatura mundial. Resultados: Um paciente apresentando fístula colo-vesical foi submetido ao tratamento laparoscópico assistido com a mão (HALS). O tempo operatório foi de 183 minutos e a alta se deu no $4^{\circ}$. dia pós-operatório. Outro paciente, portador de abscesso diverticular, submetido ao mesmo método, com tempo operatório de 145 minutos, recebeu alta no $5^{\circ}$. dia pós-operatório. Não houve morbidade nem mortalidade. Conclusão: A técnica (Hals) alia vantagens de ambos os métodos, parece ser mais rápida e segura permitindo o tratamento de diverticulite complicada. Mais estudos são necessários.
\end{abstract}

Descritores: colectomia, doença diverticular, diverticulite cólica, laparoscopia, cirurgia laparoscópica.

\section{INTRODUÇÃO}

A Doença Diverticular é uma doença benigna, de boa resposta ao tratamento clínico, instituído na mudança de hábitos alimentares, maior consumo de fibras, antibioticoterapia potente nos processos inflamatórios mais simples e uso da radiologia intervencionista nos abscessos. A indicação cirúrgica fica restrita ao insucesso da terapêutica clínica e às formas mais graves da doença, como estenoses, abscessos e perfurações.
A diverticulite aguda do cólon é a complicação mais freqüente da doença diverticular e ocorre em $30 \%$ dos pacientes, dentro dos próximos 20 anos após seu diagnóstico $(1,2)$.

A laparoscopia surgiu como técnica segura e ideal para a cirurgia do sigmóide por diminuir o tempo de internação hospitalar, e melhor resultado cosmético.Por outro lado, o método apresenta várias limitações $(3,4,5,6,7,8)$, como a ausência da sensação tátil, alguma dificuldade no controle hemorrágico, trauma na manipulação e afastamento dos órgãos. Existe,

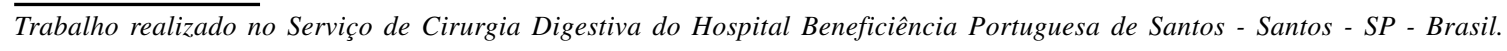

$\overline{\text { Recebido em 02/06/2006 }}$

Aceito para publicação em 16/08/2006 
ainda, a necessidade da retirada do espécime, quase sempre de grande volume, sob controle oncológico, tempo operatório prolongado e alto custo, além de necessitar equipe bem treinada para resolução dos casos (2). A obesidade constitui dificuldade para a dissecação laparoscópica (9).

No entanto, a técnica videolaparoscópica vem se tornando uma opção segura para o tratamento da doença diverticular $(1,3,10,11,12,13)$. $\mathrm{O}$ inquérito nacional brasileiro sobre cirurgia laparoscópica constata que a doença diverticular foi responsável por $20 \%$ dos procedimentos laparoscópicos. (14)

A técnica de videolaparoscopia assistida com a mão, através de novas tecnologias, como o dispositivo Lap-disk ${ }^{\circledR}$, permite a manutenção do pneumoperitônio com a utilização ou não da mão. $\mathrm{Pa}$ rece alternativa mais segura e sugere a diminuição do tempo operatório, normalmente arrastado nos procedimentos difíceis $(4,5,6,7)$. Tais dispositivos permitem a retirada da peça sob condições controladas.

A cirurgia laparoscópica assistida com a mão passou por várias fases distintas e aperfeiçoamento do material. No ínício, a colocação dos dispositivos era demorada e não permitia a continuação do procedimento de forma laparoscópica, se a mão fosse retirada (7). Novas tecnologias como o Lap Disk permitem a manutenção do pneumoperitônio, mesmo com a retirada da mão que, em alguns momentos pode ser desnecessária, ocupando espaço importante da cavidade.

A literatura ressalta a dificuldade do procedimento laparoscópico nos casos de diverticulite aguda e suas formas complicadas, sendo fator de conversão devido à dificuldade de reconhecimento das estruturas (9)

O objetivo desta publicação foi rever a literatura internacional sobre o tratamento da diverticulite aguda por laparoscopia e descrever a técnica assistida com a mão (Hals - Hand assisted laparoscopic surgery), utilizada pelos autores em dois casos. Não existem publicações nacionais sobre o tratamento da diverticulite complicada, tratada por cirurgia laparoscópica assistida com a mão, até a hora atual.

Doença diverticular e cirurgia laparoscópica. Vários autores vêm demonstrando os benefícios da técnica videolaparoscópica. Patel, numa revisão, em 2003 (15), constata que, apesar da falta de evidencia, pelo pequeno número de casos e ausência de estudos comparativos e prospectivos, as publicações sugerem benefício da cirurgia laparoscópica na diverticulite aguda. No entanto, ressaltava que a indicação ficaria restrita aos casos não complicados. $\mathrm{O}$ estudo observacional e multicêntrico da Associação Francesa de Cirurgia, comparando a cirurgia convencional e a laparoscópica na doença diverticular (13), evidencia as vantagens do método, encurtando o tempo de internação e apresentando complicações menores. Vários autores demonstraram a necessidade de conversão na presença de massas inflamatórias, fístulas, perfuração e obesidade. $(3,9,13,16,17,18)$

\section{Diverticulite aguda, formas complicadas e cirurgia laparoscópica.}

Ignjatovich, em meta-análise, em 2004, (1) conclui por vantagens na cirurgia laparoscópica da diverticulite aguda com tempo de hospitalização de 6,1 dias, deiscência anastomótica de $2,8 \%$, infecção da ferida operatória de 3\%, conversões $11,7 \%$ (0-38,9\%). Rotholtz, na Argentina (20), conclui por uma importante diminuição dos custos, comparando o procedimento laparoscópico com a cirurgia convencional. Panis (11) descreve a diminuição da taxa de conversão após os primeiros 29 casos de diverticulite aguda (de $24 \%$ para $14 \%$ ), tempo operatório igual à cirurgia aberta com retorno do trânsito e tempo de hospitalização menores na cirurgia laparoscópica. Sher (22), na Cleveland Clinic, confirma a diminuição do tempo de internação e do íleo pós-operatório.

\section{Diverticulite aguda e Cirurgia Laparoscópica assistida com a mão.}

Bartus et al (6) comparam 146 casos de diverticulite não complicada com 36 casos de fístula diverticular, operados por cirurgia laparoscópica assistida com a mão com $5 \%$ e $25 \%$ de conversão e tempo operatório de 176 e 220 minutos para cada grupo. O tempo de internação foi de 4,4 e 6,2 dias.Não houve mortalidade.

Lee et al. (4) compararam 21 casos de diverticulite aguda tratados por cirurgia laparoscópica e 21 casos tratados pela técnica Hals. Relataram uma diminuição do tempo operatório ( $255 \pm 18$ versus $177 \pm 34$ minutos), não havendo diferenças estatisticamente significantes nas taxas de conversão, tempo de internação, complicações ou perdas sanguíneas. No entanto, todos os casos de cirurgia laparoscópica convertidos $(3-14 \%)$ foram tratados com uma incisão Pfannenstiel, parecendo razoável, para o autor, iniciar a cirurgia com a incisão e utilizá-la desde o inicio com a técnica Hals. 


\section{TÉCNICA}

CASO 1: GBS, 47 anos, sexo masculino, branco, com história de pneumatúria intermitente há aproximadamente 1 mês. Não apresentava outras queixas. Uma tomografia de abdome evidenciava massa sigmoideana aderida à bexiga e gás no interior da mesma, quadro compatível com diverticulite complicada por fístula sigmoido-vesical. Após preparo do cólon com manitol 10\%, 1 litro na véspera, antibioticoterapia profilática com cefoxitina $1,0 \mathrm{~g} \mathrm{EV}$ na indução anestésica e tromboprofilaxia com enoxiparina 20 ui. $\mathrm{SC}$, o paciente foi colocado em posição de LLoyd Davies modificada, fixo à mesa e submetido à videolaparoscopia aberta que evidenciou massa inflamatória sigmoideana aderida à bexiga. Os trocartes foram colocados em posição habitual para retosigmoidectomia laparoscópica (fig.1). Após a liberação da goteira parieto-cólica esquerda até o ângulo esplênico, iniciou-se a liberação da massa, próximo à bexiga, constando-se uma importante fixação à parede lateral esquerda. Realizamos, assim, uma incisão transversa tipo Pfannenstiel de $7 \mathrm{~cm}$ para a colocação do dispositivo Lap Disk®. Figura 1.

A introdução da mão permitiu a liberação da parede lateral esquerda, firmemente aderida ao ureter. Uma vez liberado, a face medial do mesocolo sigmóide foi aberta e seu pedículo ligado através de seladeira de

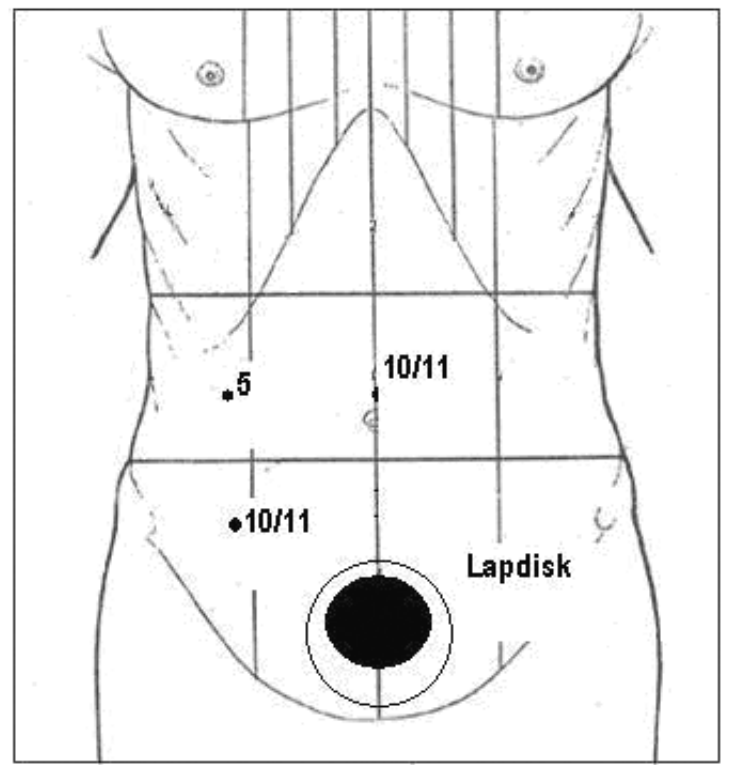

Figura 1 - Disposição dos trocartes para Retosigmoidectomia assistida com a mão. vasos (Ligasure). O cólon foi exteriorizado através do dispositivo e o reto fechado através de grampeamento linear convencional (Figura 2).

Realizamos uma descendente-retoanastomose com grampeador circular $31 \mathrm{~mm}$ introduzido pelo anus. A bexiga foi enchida com azul de metileno, não se constatando fístulas. A cavidade foi drenada com dreno siliconizado. O tempo operatório foi de $182 \mathrm{~min}$. O paciente foi realimentado no segundo dia de pós-operatório, recebendo alta hospitalar no quarto dia.

Caso 2: ACJ, 41 anos, sexo masculino, branco, obeso foi internado em situação de emergência com diagnóstico de diverticulite aguda em segundo ataque, submetido a antibióticoterapia por 10 dias sem melhora importante. Evoluiu com abscesso diverticular à tomografia.

Assim, foi submetido a procedimento idêntico ao descrito acima que revelou um abscesso diverticular bloqueado por alças de delgado. O uso da mão permitiu uma dissecação digital das alças de delgado aderidas ao sigmóide e a liberação do mesmo com facilidade.O tempo operatório foi de 145 minutos, o paciente foi realimentado no $3^{\circ}$ pós-operatório e recebeu alta no $5^{\circ}$ dia. Evolui sem morbidade

\section{DISCUSSÃO}

A cirurgia laparoscópica tem aparecido, cada vez mais, como uma opção de cirurgia de urgência para as mais variadas patologias, como apendicite aguda, colecistite aguda, patologias anexiais, diverticulite aguda, entre outros. $(10,11,12)$

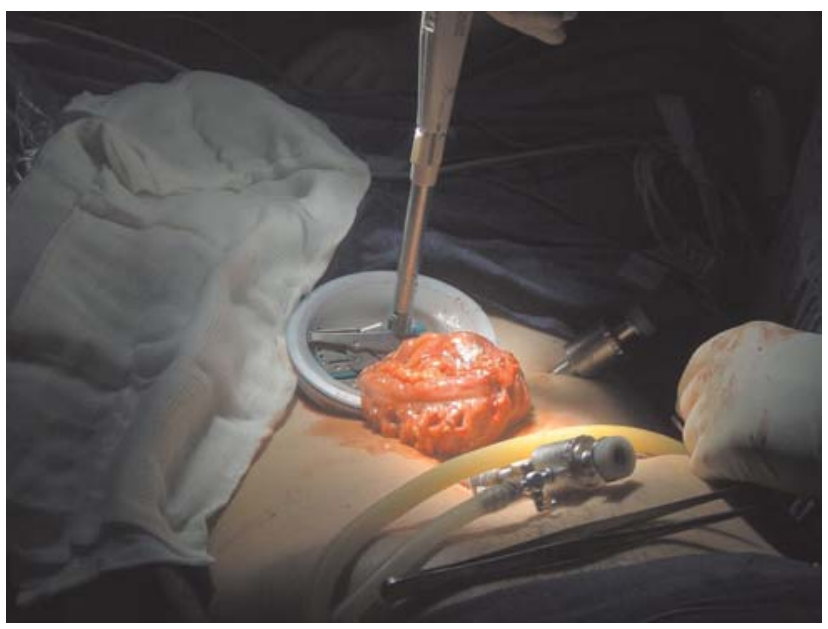

Figura 2 - Fechamento do reto com grampeador linear. 
Desde 1991, quando o primeiro caso de colectomia laparoscópica foi divulgado, esta técnica tem se disseminado cada vez mais na cirurgia colo-retal. Enquanto restem algumas dúvidas quanto ao tratamento das malignidades, na doença benigna o benefício está bem estabelecido, apresentando eficácia similar ao tratamento convencional, encurtando o tempo de internação e o retorno às atividades. $\mathrm{O}$ custo tem se demonstrado menor na cirurgia laparoscópica (20).Este método ainda não está sendo amplamente utilizado porque apresenta algumas dificuldades técnicas e exige equipe bem treinada (6).

Atualmente está bem definida a indicação da cirurgia laparoscópica para sigmoidectomias eletivas por diverticulite, ressecções íleo-cecais por Doença de Crohn, ressecção de pólipos colônicos volumosos, entre outras. (7)

Tradicionalmente, fístulas colo-vesicais eram consideradas contra-indicação para colectomias laparoscópicas, mas, com o advento da laparoscopia assistida com a mão, tem-se evidenciado a possibilidade de realizar adequadamente esse tratamento (6).

Bartus et al. relatam que é verdadeiramente mais difícil operar esses casos por laparoscopia e que a cirurgia é mais demorada e complicada, além do índice de conversão ser maior quando comparado às cirurgias de diverticulite não complicada. Porém, o doente se beneficia intensamente, já que o tempo de internação e a evolução pós-operatória são similares aos da cirurgia para doença não complicada. Enfim, os autores sugerem, antecipadamente, que a cirurgia laparoscópica assistida com a mão será o tratamento padrão da diverticulite complicada.

Tagarona (23) compara o processo inflamatório em 27 procedimentos HALS com 27 procedimen- tos laparoscópicos, demonstrando aumento de Interleucina 6 e proteína $C$ reativa na técnica HALS. Os escassos estudos comparativos com cirurgia laparoscópica não demonstram vantagens nem desvantagens quanto ao tempo de internação, morbidade $(4,24,25,26)$. Alguns constatam diminuição no tempo cirúrgico. $(4,26)$

\section{CONCLUSÕES}

São muitas as vantagens da cirurgia laparoscópica quando comparada à cirurgia aberta no tratamento das afecções benignas. As desvantagens se devem principalmente às conversões, morbidade $\mathrm{e}$ tempo operatório, o que torna a experiência do cirurgião um importante fator para o sucesso da cirurgia, lembrando que a curva de aprendizado é longa.

$\mathrm{Na}$ análise crítica que se faz do instrumental laparoscópico, não é possível reproduzir os movimentos da mão sem assistência robotizada, $(16,27)$ o que torna difícil a mobilização de massas e a dissecação de graves processos inflamatórios.

A técnica HALS alia a excelente imagem da cirurgia laparoscópica com a propriocepção e manipulação delicadas que a mão é capaz de fazer. $(4,6,7,8)$ Parece encurtar o tempo cirúrgico, além de proporcionar maior ousadia no tratamento de casos difíceis. $\mathrm{O}$ método utilizado correspondeu a 5\% da experiência do autor em colectomias laparoscópicas; no entanto, nos pareceu mais fácil e mais seguro $(6,23,24,25)$.

A técnica HALS deve fazer parte do arsenal da cirurgia laparoscópica, especialmente nos casos difíceis. No entanto, a literatura sobre a técnica ainda é pequena, e são necessários mais estudos prospectivos, com maior número de casos.

\footnotetext{
ABSTRACT: The laparoscopic surgery for diverticular disease has become a very good alternative. The hand-assisted allows combining the advantages of conventional surgery such as tactile perception, masses mobilization without trauma, vascular control with the magnifying laparoscopic view. Difficult cases of diverticulitis can be treated with the hand-assisted approach. The authors revised the literature and describe the technique utilized in 2 cases of complicated diverticulitis. Results: Two patients were operated on a hand-assisted approach. One had fistula with the bladder. The operative time was 183 minutes and hospitalization period about 4 days. Other had a sigmoid abscess. The operative time was 145 minutes and hospitalization period about 5 days. There were no morbidities. Conclusion: The hand-assisted approach allies advantages of both methods and it seems to be safer and fast allowing laparoscopic techniques for complicated diverticulitis. More studies are necessary.
}

Key words: Colectomy, hand-assisted laparoscopic colectomy, diverticular disease, diverticulitis. 


\section{REFERÊNCIAS}

1. Ignjatovic D, Zivanovic V, Vasic G, Ilic I. Meta-analysis on minimally invasive surgical therapy of sigmoid diverticulitis. Acta Chir Iogusl 2004; 51(3): 25-8.

2. Vuillemuier H, Nordback P, Givel JC. Treatment of acute diverticulitis. Rev Med Suisse 2005; 1(24):1600-3.

3. Scwander O, Farke S, Fischer F, Eckman C, Schiedeck THK, Bruch HP. Laparoscopic colectomy for recurrent and complicated diverticulitis: a prospective study of 396 patients. Langenbecks Arch Surg. 2004; 389: 97-103.

4. Lee SW, Yoo J, Duvony N, Sonoda T, Milsom JW. Laparoscopic vs.Hand-assisted laparoscopic Sigmoidectomy for Diverticulitis. Dis Colon Rectum 2006; 49:464-9.

5. Darzi A. Hand-assisted laparoscopic surgery. Surg Endosc 2000;14: 999-1004.

6. Bartus CM. Colovesical fistula: not a contraindicacion to elective laparoscopic colectomy. Dis Colon Rectum 2005: 48(2): 233-6.

7. Campos FGCM. Cirurgia laparoscópica assistida com a mão. Indicações e resultados preliminares em procedimentos coloretais. Rev Bras Coloproct 2005; 25(1): 94-101.

8. Pupo Neto JÁ, Lacombe D. laparoscopic hand-assisted surgery: comparative randomized study with laparotomy. Rev Bras Videocir 2003; 1:60-70.

9. Tekkis PP, Senagore AJ, Delaney CP. Conversio rates in laparoscopic colorectal surgery. A predictive model with 1253 patients. Surg Endosc 2005;19: 47-54.

10. Panis Y. Laparoscopic sigmoid resection for diverticulitis: is learning phase associated with increased morbidity? Ann Chir 2003; 128(2):81-7.

11. Panis Y. Laparoscopic surgery for benign colorectal disease. J Chir 2000; 137(5):261-7.

12. Perri SG. Laparoscopy in abdominal emergencies. Indications and limitations. Chir Tal 2002; 54(2):165-78.

13. Alves A, Panis Y, Slim K, Heyd B, Kwiatkowski F, Mantion G. French multicentre prospective observational study of laparoscopic versus open colectomy for sigmoid diverticular disease. Br J Surg 2005;92:1520-5.

14. Campos FGCM. Cirurgia Laparoscópica Colorretal. Resultados do Inquérito Nacional Brasileiro. Rev Bras Coloproct 2001; 21(3): 135-43.

15. Patel NA, Bergamschi R. Laparoscopy for diverticulitis. Semin Laparosc Surg 2003;10(4):177-83.

16. Woeste G, Bechstein WO, Wulstein Cl. Does telerobotic assistance improve laparoscopic colorectal surgery? Int J Colorectal Dis 2005;20(3): 253-7.
17. Hidebrandt U, Kreissler-Haag D, Lindermann W. Laparoscopic-assisted colorectal resections: morbidity, conversions, outcomes of a decade. Zentralbl Chir 2001; 126(4): 323-32.

18. Perniceni T, Burdy G, Gayet B, Dubois F, Boudet MJ, Levrard $\mathrm{H}$. Results of elective segmental colectomy done with laparoscopy for complicated diverticulosis. Gatroenterol Clin Biol 2000; 24(2): 189-92.

19. Carbajo CMA, Mertin Del Olmo JC, Blanco Alvarez JL, Martin Acebes F, De la Cuesta de la Llave C, Atienza SR, et al. Acute diverticulitis and diverticular disease of the colon: a safe indication for laparoscopic surgery. Rev Esp Enferm Dig 2000; 92(11):718-25.

20. Rotholtz NA, Estruch C, Bosio RM, Gorodner AA, Peczan CE, Mezzadri NA. La sigmoidectomia laparoscópica por enfermedad diverticular es costo efectiva? Ver Argent Cir 2005;88(1-2):63-9

21. Chen HH, Wexner SD, Iroatulam AJ, Pikarsky AJ, Alabaz O, Nogueira JJ, et al. Laparoscopic colectomy compares favorably with colectomy by laparotomy for reduction of postoperative ileus. Dis Colon Rectum 2000; 43(1): 61-5.

22. Sher ME, Agachan F, Bourtoul M, Nogueras JJ,Weiss EG, Wexner SD. Laparoscopic surgery for diverticulitis. Surg Endosc 1997; 11(3): 264-7.

23. Tagarona EM, Gracia E, Garriga J, Martinez-Bru C, Cortes M, Boluda $\mathrm{R}$, et al. Prospective randomized trial comparing conventional laparoscopic colectomy with hand-assisted laparoscopic colectomy: aplicability, immediate clinical outcome, inflammatory response and cost. Surg Endosc 2002;16(20):234-9.

24. Hand-assisted laparoscopic surgery vs standard laparoscopic surgery for colorectal disease: a prospective randomized trial. Hals Study Group. Surg Endosc 2000;14(10):896-901.

25. Cobb WS, Lokey JS, Schwab DP, Crockett JA, Rex JC, Robbins JA. Hand-assisted laparoscopic colectomy: a single-institution experience. Am Surg 2003; 69(7):578-80.

26. Rivadeneira DE, Marcello PW, Roberts PL, Rusin LC, Murray JJ, Coller JÁ, et al. Benefits of hand-assisted laparoscopic restorative proctocolectomy: a comparative study. Dis Colon Rectum 2004; 47(8):1371-6.

27. Talamini MA. Laparoscopy for benign disease: robotics. Semin Laparosc Surg 2003; 10(4): 203-8.

\section{Endereço para correspondência: GUINES ANTUNES ALVAREZ}

Rua Marechal José Olintho de Carvalho, 9 11.070 -210 - Santos (SP)

E-mail: guinesalvarez@uol.com.br 